\title{
Cervical oesophageal perforation secondary to food consumption in a well-appearing patient
}

\author{
Alexander L Schneider, Katherine E Hicks, Akihiro J Matsuoka
}

Department of Otolaryngology Head and Neck Surgery, Northwestern University Feinberg School of Medicine, Chicago, Illinois, USA

\section{Correspondence to} Dr Alexander L Schneider, alexander.schneider@ northwestern.edu

Accepted 7 November 2017

\section{SUMMARY}

A 71-year-old woman presented to the emergency department 8 days after ingesting fish with mild neck pain but otherwise demonstrated no signs of infection. $X$-rays were negative but $C T$ imaging demonstrated a curvilinear radiodense object extending from the posterior cervical oesophagus through the right thyroid lobe terminating in the neck just a few millimetres from the external carotid artery. Rigid oesophagoscopy and direct laryngoscopy were negative and the neck was explored for the foreign body, which ultimately was encountered after a painstaking dissection of the right neck that included skeletonisation of the recurrent laryngeal nerve. Her postoperative recovery was uneventful and after a 3-day course of intravenous antibiotics she was discharged on oral antibiotics, in good condition and tolerating a soft diet.

\section{BACKGROUND}

This case is important because it touches on an uncommon presentation of a common otolaryngological consultation: the ingested upper aerodigestive tract (UADT) foreign body (FB). In the case presented, our patient had gone over 1 week with an ingested FB that migrated extraluminally through the wall of her oesophagus to lodge in the soft tissues of the neck. Despite the presumed cervical oesophageal perforation, our patient displayed no clinical nor radiological signs of an infectious process. Furthermore, despite relatively precise CT localisation of the $\mathrm{FB}$, the authors had difficulty finding the bone and only did so after skeletonising a vital nervous structure in the neck. Thus, we believe that this case is important because it demonstrates the following: the unique ability of the
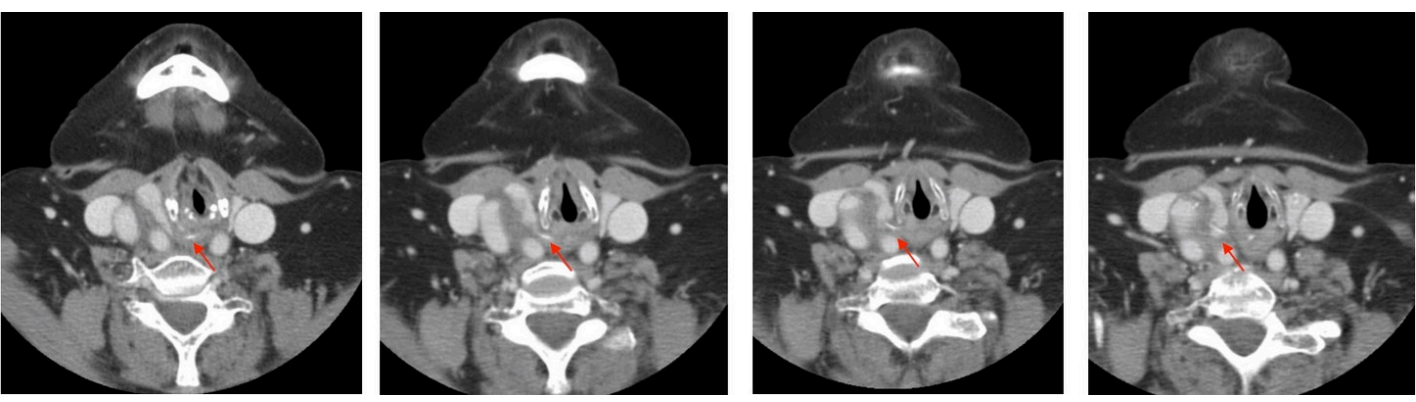

Figure 1 Contrast-enhanced, axial plane CT demonstrating the curvilinear radiodense foreign object coursing from the posterior cervical oesophagus anterolaterally to the patient's right, piercing the thyroid gland and terminating near the external carotid artery.

cervical fascial planes to impede infection, the importance in pursuing CT imaging in working up an oesophageal FB, and the degree to which the surgeon must be willing to dissect delicate structures of the neck in a FB that has migrated through the oesophagus into the soft tissues of the neck.

\section{CASE PRESENTATION}

A 71-year-old Hispanic woman presented to the emergency department 8 days after ingesting fish and swallowing a fishbone. Her main complaints were mild odynophagia and mild right-sided neck pain. She was afebrile with normal vital signs. Physical exam demonstrated a soft and flat neck without any crepitus or palpable fluid collections. She had full neck range of motion with very mild pain with neck rotation. She was noted to have poor neck extension at baseline secondary to body habitus. Her leucocyte count was $12.4 \times 10^{9} / \mathrm{L}$; all other laboratory parameters were within normal limits.

\section{INVESTIGATIONS}

Bedside flexible fibre-optic laryngoscopy demonstrated mild to moderate oedema and erythema of the right arytenoid; however, no FB or mucosal laceration was visualised.

Formal AP and lateral neck X-rays were unremarkable; however, a contrast-enhanced CT scan demonstrated a 3-centimetre long curvilinear hyperdense mass coursing from the posterior cervical oesophagus, through the superior aspect of the right thyroid lobe, and terminating in the soft tissues of the right neck, just a few millimetres away from the right external carotid artery. Of note, there was no fluid collection, fat 


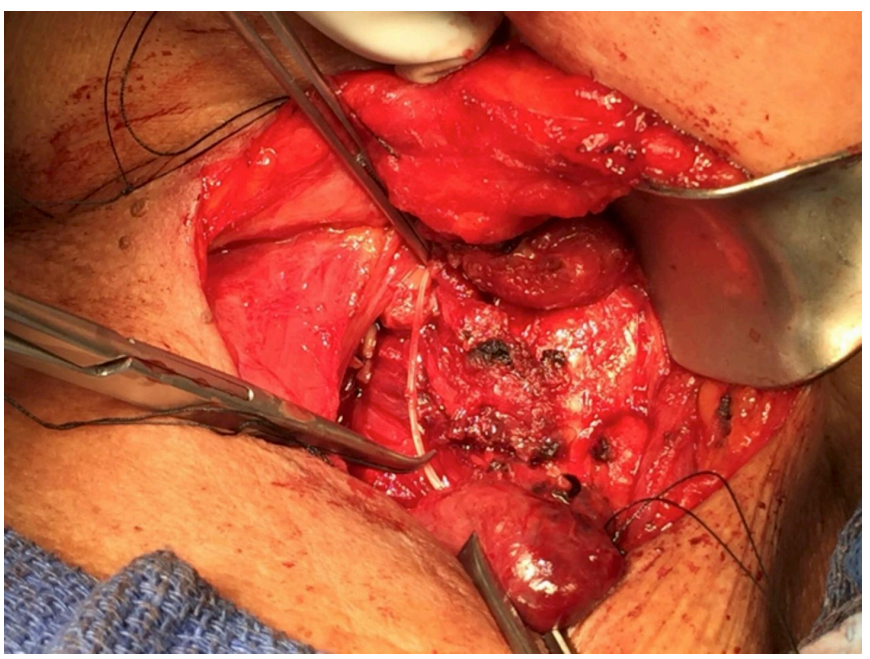

Figure 2 The fishbone can be seen adjacent to the right tubercle of Zuckerkandl.

stranding or gas seen on her CT scan. Representative images are shown in figure 1

\section{TREATMENT}

Although her clinical exam and X-rays were reassuring, her CT scan suggested the presence of a transoesophageal FB and demonstrated its proximity to major vasculature of the head and neck. As such, the patient was scheduled

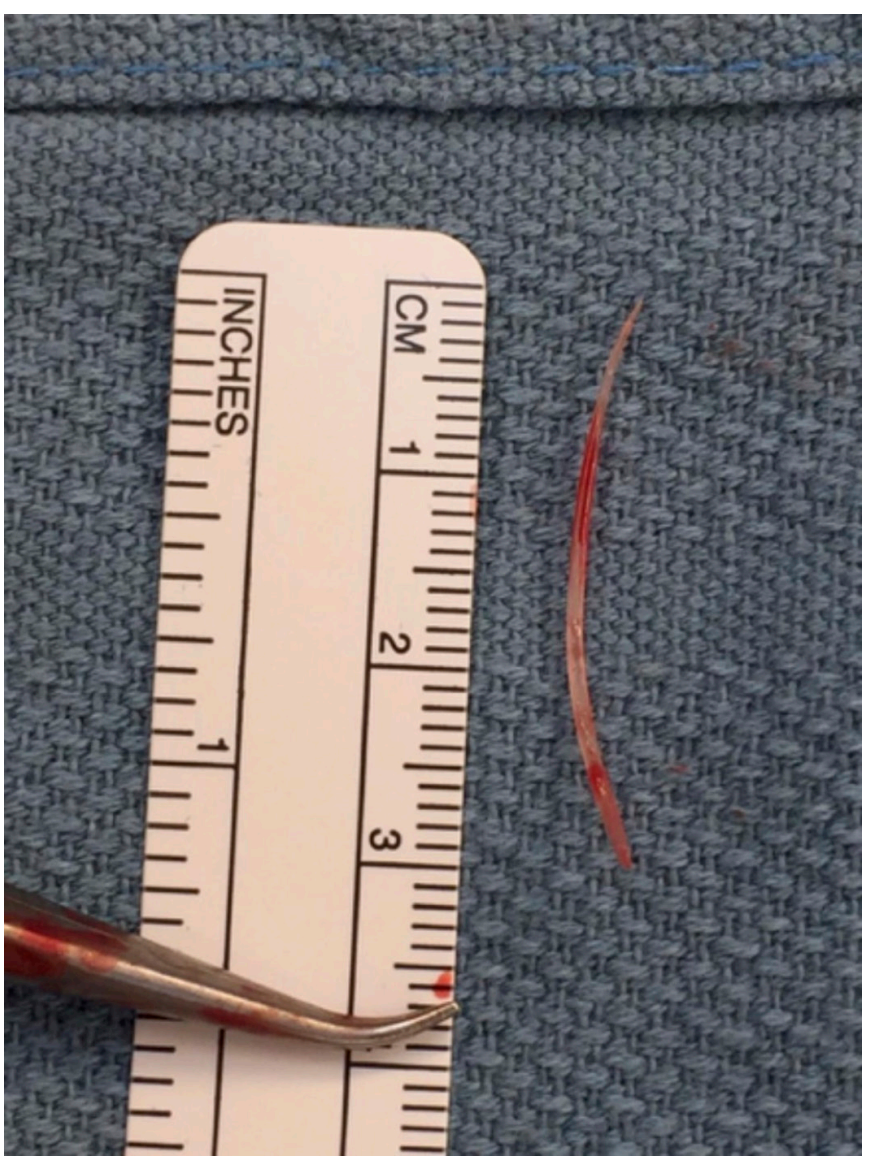

Figure 3 The fishbone after removal, measuring over $3 \mathrm{~cm}$ by less than $0.5 \mathrm{~cm}$. for panendoscopy and possible transcervical approach for removal of the FB. During endoscopyendoscopy, oesophageal secretions were foul smelling; however, no frank purulence was encountered, and the FB was unable to be visualised. The decision was then made to perform a transcervical approach, which was done via a horizontal thyroidectomy-type incision offset to the right side of her neck. As suggested by her physical exam, we were unable to achieve favourable neck extension despite having a shoulder roll in place, making exposure and exploration more challenging. After taking the incision through skin, subcutaneous tissues and the platysma muscle, subplatysmal flaps were elevated superiorly to the thyroid notch and inferiorly to the clavicle. To optimise exposure, the strap muscles were divided using cautery and retracted laterally, thus exposing the right thyroid lobe.

The dissection was carried medial to the sternocleidomastoid muscle to expose the carotid sheath, and then the medial aspect of the external carotid artery was skeletonised. The dissection was carried posteriorly to the level of the prevertebral fascia. The lobe was mobilised laterally and superiorly, as would be done for a standard lobectomy, and these areas were carefully explored for evidence of FB. Despite the above manoeuvres, we were unable to locate the fishbone. An intraoperative X-ray was taken to assist with localisation, but no calcified material was visualised on plain film.

CT scan images were reviewed again intraoperatively, and it appeared that the $\mathrm{FB}$ was in close proximity to the cricothyroid joint. Thus, the decision was made to dissect out the recurrent laryngeal nerve, and the tubercle of Zuckerkandl was mobilised. Immediately deep to the tubercle and just superficial to the recurrent laryngeal nerve, the fishbone was encountered and was removed in its entirety (figures 2 and 3). There was mild oedema within the soft tissues of the neck; however, there was no purulent fluid identified at any point in the dissection. The wound was then extensively irrigated, a suction drain was placed, and the neck was closed in layers. Postoperatively, the patient was given intravenous ampicillin-sulbactam for 3 days, then converted to oral amoxicillin-clavulanate. She did well, tolerated a diet with no signs of infection and was discharged home in good condition on postoperative day 3 following drain removal.

\section{OUTCOME AND FOLLOW-UP}

The fishbone was removed in its entirety and can be seen in figures 2 and 3. Postoperatively, the patient was given intravenous ampicillin-sulbactam for 3 days, then converted to oral amoxicillin-clavulanate. She was kept nil per os for the first 24 hours after surgery. Following this, she did well, tolerated a diet with no signs of infection, and was discharged home in good condition on postoperative day 3 following drain removal. She then opted to pursue the remainder of her care at a facility nearer to her home.

\section{DISCUSSION}

Ingested UADT FBs are prevalent in otolaryngological practice, and the majority remain intraluminal ${ }^{1}$ and are able to be removed endoscopically. ${ }^{2}$ The most common locations for fishbones to become lodged are the palatine tonsils, followed by base of tongue, vallecula and pyriform sinuses. ${ }^{3}$ Many of these cases can be managed with direct or endoscopic visualisation with subsequent removal of FB. Oesophageal FBs 
are relatively rare, and FBs that migrate through the oesophageal wall and lodge in the soft tissues of the neck occur very infrequently. In these instances, direct rigid or flexible oesophagoscopy may be attempted; however, the surgeon must also be prepared for possible transcervical exploration for removal of FB.

The rate of oesophageal perforation in the setting of an ingested oesophageal FB has been estimated to be $2 \%,{ }^{4}$ though there was no distinction made as to whether the FBs were completely extraluminal as opposed to partially intraluminal. While rare, the resultant sequelae of an oesophageal perforation secondary to ingested FB include mediastinitis, carotid rupture, deep neck space abscesses and even cardiac tamponade, each of which portends significant morbidity. ${ }^{5-7}$

It is interesting to note that despite the fact that our patient presented greater than 1 week after her fishbone ingestion with radiographic evidence of extraluminal migration, she had an overall benign physical exam and no evidence of abscess or abnormal air on her CT scan. Additionally, she was afebrile, haemodynamically stable, and had not developed any of the aforementioned devastating complications. In the thoracic surgery literature, it has been postulated that cervical oesophageal perforations are often less severe and more easily treated than intrathoracic or intraabdominal perforations. ${ }^{8}$ This phenomenon is thought to be due to the tightly adherent fascial planes of the neck, limiting the spread of oesophageal contamination to neighbouring spaces, particularly the mediastinum. ${ }^{9}$

The relative resistance of the fascial planes to the spread of infection in cases of cervical oesophageal contamination may be responsible for the variable manner in which cervical oesophageal perforations may present, which can further delay treatment and diagnosis. In the presence of a convincing clinical history for fishbone ingestion but negative X-rays, the clinician may proceed directly to the operating suite for endoscopy, but should be ready to obtain a CT scan to both rule in/rule out the presence of the fishbone as well as assist with surgical planning for open neck exploration. While X-rays are often still the first diagnostic modality used given widespread availability, the published sensitivity of X-ray detection for fishbones ranges from just $27 \%$ to $57 \% .{ }^{10} \mathrm{CT}$ scan, on the other hand, has been shown to have a sensitivity of $100 \%$ and specificity of $93.7 \% .{ }^{11}$ In our case, despite having a preoperative CT scan to localise the fishbone, we were initially unable to find the object in the neck, and formal intraoperative X-rays also did not detect the bone. The authors suspect that the difficulty in identifying the fishbone intraoperatively partially stems from the optimisation of neck extension in the operating room, compared with suboptimal positioning during imaging. Thus, the surgeon must maintain a high index of suspicion for possible ingested fishbone and have a low threshold to obtain CT imaging in the face of negative $\mathrm{X}$-rays and endoscopy. On open neck exploration, the surgeon must be willing to carefully and thoroughly explore delicate structures in order to remove the FB in question. The surgeon should also keep in mind that changes in patient positioning during imaging versus in the operating room may have a noticeable effect on location of FB.

\section{Learning points}

- Upper aerodigestive tract foreign bodies (FBs) are common in the otolaryngologist's practice. When the FB in question is a fishbone, the practitioner must have a high index of suspicion to pursue advanced imaging, particularly in the case of negative $\mathrm{X}$-rays and endoscopy.

- Despite the morbidity associated with oesophageal perforations, the well-defined and tightly adherent fascial planes of the cervical neck may play a role in impeding the development or spread of infection in cases of cervical oesophageal perforation.

- CT scan may be useful for localisation and surgical planning; however, the surgeon must account for changes in patient body position between that of the CT scan and that of the operating room. Further, after open neck exploration the surgeon must be willing to dissect fine anatomical structures in order to remove the FB in question.

Contributors AS, $\mathrm{KH}$ and $\mathrm{AM}$ each made substantial contributions to the conception or design of the work, or the acquisition, analysis or interpretation of data; were responsible for drafting the work or revising it critically for important intellectual content; provided final approval of the version published; agree to be accountable for all aspects of the work; agree to ensure that questions related to the accuracy or integrity of any part of the work are appropriately investigated and resolved.

Competing interests None declared.

Patient consent Obtained.

Provenance and peer review Not commissioned; externally peer reviewed.

(C) BMJ Publishing Group Ltd (unless otherwise stated in the text of the article) 2017. All rights reserved. No commercial use is permitted unless otherwise expressly granted.

\section{REFERENCES}

1 Joshi AA, Bradoo RA. A foreign body in the pharynx migrating through the internal jugular vein. Am J Otolaryngol 2003;24:89-91.

2 Megwalu UC. Migration of an ingested fish bone into the paraglottic space. J Laryngol Otol 2016;130:973-4.

3 Woo SH, Kim KH. Proposal for methods of diagnosis of fish bone foreign body in the Esophagus. Laryngoscope 2015;125:2472-5

4 Sung SH, Jeon SW, Son HS, et al. Factors predictive of risk for complications in patients with oesophageal foreign bodies. Dig Liver Dis 2011;43:632-5.

5 Chen CY, Peng JP. Esophageal fish bone migration induced thyroid abscess: case report and review of the literature. Am J Otolaryngol 2011;32:253-5.

6 Chen HH, Ruan LX, Zhou SH, et al. The utility of repeated computed tomography to track a foreign body penetrating the esophagus to the level of the thyroid gland. Oral Radiol 2014;30:196-202.

7 Sharland MG, McCaughan BC. Perforation of the esophagus by a fish bone leading to cardiac tamponade. Ann Thorac Surg 1993;56:969-71.

8 Brinster CJ, Singhal S, Lee L, et al. Evolving options in the management of esophageal perforation. Ann Thorac Surg 2004;77:1475-83.

9 Jones WG, Ginsberg RJ. Esophageal perforation: a continuing challenge. Ann Thorac Surg 1992;53:534-43.

10 Evans RM, Ahuja A, Rhys Williams S, et al. The lateral neck radiograph in suspected impacted fish bones--does it have a role? Clin Radiol 1992;46:121-3.

11 Kim JP, Kwon OJ, Shim HS, et al. Analysis of Clinical Feature and Management of Fish Bone Ingestion of Upper Gastrointestinal Tract. Clin Exp Otorhinolaryngol 2015;8:261-7. 
Copyright 2017 BMJ Publishing Group. All rights reserved. For permission to reuse any of this content visit http://group.bmj.com/group/rights-licensing/permissions.

BMJ Case Report Fellows may re-use this article for personal use and teaching without any further permission.

Become a Fellow of BMJ Case Reports today and you can:

- Submit as many cases as you like

- Enjoy fast sympathetic peer review and rapid publication of accepted articles

- Access all the published articles

- Re-use any of the published material for personal use and teaching without further permission

For information on Institutional Fellowships contact consortiasales@bmjgroup.com

Visit casereports.bmj.com for more articles like this and to become a Fellow 